\title{
Imagerie nanométrique femtoseconde par diffraction $X$ cohérente
}

\author{
Willem BOUTU et Hamed MERDJI \\ Service des Photons, Atomes et Molécules, CEA-Saclay, 91191 Gif-sur-Yvette Cedex \\ willem.boutu@cea.fr, hamed.merdji@cea.fr
}

Dans de nombreux domaines de la science, nous cherchons à voir la matière évoluer en temps réel. Imager des objets individuels en combinant des résolutions spatiales de l'ordre du nanomètre $\left(10^{-9} \mathrm{~m}\right)$ et temporelles de l'ordre de la femtoseconde $\left(1 \mathrm{fs}=10^{-15} \mathrm{~s}\right.$ ) représente donc un enjeu important. Très récemment, le développement des sources $X$ cohérentes récentes de type « laser » nous permet d'atteindre des résolutions jusque-là inégalées. C'est dans ce contexte que se situent les recherches menées par le laboratoire Attophysique du CEA Saclay: développer de nouvelles sources de lumière ultra-brèves, contrôlées, dans le domaine des rayons $X$, afin d'imager des phénomènes physiques, chimique, biologiques, à une échelle spatiale atomique/nanométrique avec des résolutions temporelles attoseconde/femtoseconde.

\section{Vers les résolutions ultimes}

Imager des objets individuels, à l'échelle nanométrique avec des résolutions temporelles ultrabrèves, représente, de nos jours, un challenge fascinant qui va au-delà des techniques établies à ce jour. Pour obtenir une image d'un objet, il suffit usuellement de l'éclairer et d'enregistrer la lumière diffusée qui parvient à un détecteur. Si l'image est formée à l'aide d'un objectif, I'optique utilisée impose de nombreuses limitations (résolution, aberrations...).

Pour atteindre les résolutions ultimes, spatialement avec des rayons $X$ (résolution fonction de la longueur d'onde du rayonnement utilisée) et temporellement avec des impulsions ultra-brèves (fonction de la durée du «flash»), des techniques possibles, sans optique, sont la diffraction X cohérente (Miao et al., 1999) ou encore I'holographie $X$ par transformée de Fourier (McNulty et al., 1992). Les techniques d'imagerie sans lentilles ont été développées et appliquées largement dans le domaine optique.

Ces techniques profitent d'un faisceau cohérent pour obtenir une figure de diffraction de l'objet et pour reconstruire l'objet à partir de la mesure de l'intensité de diffraction de l'objet. Théoriquement, la qualité de reconstruction est déterminée par la limite de diffraction.

\section{Des sources $X$ cohérentes aux lasers à électrons libres}

Les études pionnières dans le domaine des rayons ont été réalisées principalement sur synchrotron et ce n'est que très récemment que les sources $X$ cohérentes ultrabrèves de type laser ont permis de réaliser des avancées considérables. Les premières études de Coherent Diffractive Imaging ultra-brève ont permis d'imager des objets nanométriques isolés, en utilisant une impulsion XUV femtoseconde unique (durée de l'ordre de 20 fs), délivrée par le laser à électrons libres FLASH de Hambourg (Chapman et al., 2006). Ces résultats peuvent révolutionner les scien ces de l'imagerie en permettant d'imager des systèmes nanométriques, des macromolécules, des virus isolés, sans avoir recours à la cristallisation. Ce n'est que très récemment que l'image d'un virus unique a ainsi pu être obtenue sur le laser à électrons libres de Stanford (Seibert et al., 2011).

Avec des impulsions $X$ cohérentes ultrabrèves, il est de plus possible de filmer la dynamique de systèmes complexes aussi bien en biologie, en physique qu'en chimie. Cette perspective a fortement motivé la construction des lasers à électrons libres en opération ou en projet dans le monde. Cependant le coût de ces machines ne les rend accessibles qu'à quelques pays, ce qui limite la dissémination de cette recherche. Il est donc important de disposer pour ces applications de sources de laboratoire.

\section{Optimiser la génération d'harmoniques}

La génération d'harmoniques d'ordre élevé possède toutes les propriétés nécessaires aux études résolues en temps par l'imagerie cohérente (cohérence spatiale, impulsions femtosecondes voire attosecondes, large couverture spectrale...). Le nombre de photons délivrés a été une des limitations pour la réalisation d'applications. Aussi l'optimisation de l'efficacité de génération a été et reste un enjeu majeur. Depuis quelques années, de nombreux efforts ont permis de réaliser une ligne de lumière fiable et très intense. Elle délivre des énergies de l'ordre du microjoule avec d'excellentes propriétés spatiales et temporelles. Pour ce faire, nous focalisons environ $10 \mathrm{~mJ}$ d'énergie laser dans une cellule de gaz (argon ou néon) de grandes dimensions (de l'ordre de $6 \mathrm{~cm}$ de longueur) 
grâce à une lentille de plus de $5 \mathrm{~m}$ de longueur focale. Ceci permet d'augmenter le volume focal, et donc le nombre d'émetteurs potentiels, mais aussi de créer de meilleures conditions pour la construction macroscopique cohérente du faisceau XUV grâce à une évolution lente et douce du champ laser. L'utilisation d'optiques de focalisation avec un dépôt multicouches adapté ainsi que de filtres métalliques de haute qualité nous garantit $10^{9}$ photons par impulsion (de $20 \mathrm{fs}$ ) au niveau de l'échantillon pour une longueur d'onde de $32 \mathrm{~nm}$ et $10^{8}$ photons par impulsion à $20 \mathrm{~nm}$. Ceci nous a permis de démontrer pour la première fois la faisabilité de l'imagerie cohérente simple tir sur une source de laboratoire (Ravasio et al., 2009). Puis nous avons exploré un nouveau schéma holographique, plus robuste, qui permet également d'atteindre des résolutions nanométrique et femtoseconde (Gauthier et al., 2010).

Ainsi, la source harmonique, compacte et dotée de propriétés spécifiques, devient complémentaire des grandes installations comme le rayonnement synchrotron et le laser à électrons libres. Avec des durées attoseconde à femtoseconde, des études dynamiques de l'échelle moléculaire à des échelles mésoscopiques peuvent enfin être envisagées sur des installations de taille et de coût modeste.

\section{L'imagerie par diffraction X cohérente}

Lorsqu'un faisceau de lumière cohérente illumine un objet, la figure de diffraction dans le champ lointain est la transformée de Fourier des modifications, en amplitude et/ou en phase, de l'éclairage par l'objet. C'est la détection et l'analyse du champ électromagnétique diffracté par l'objet en champ lointain qui doit permettre de retrouver l'information utile. En microscopie visible, on utilise en général une lentille afin de réaliser la transformée inverse permettant de remonter à l'image de l'objet. Cependant quand on utilise du rayonnement $X$ cette procédure ne peut pas être suivie parce qu'il est très difficile de réaliser des optiques réfractives dans ce domaine spectral. C'est pour cela que l'on s'intéresse à la technique d'imagerie par diffraction cohérente qui ne nécessite pas de système optique (technique communément appelée lensless imaging).

Développée dans un premier temps sur les sources synchrotrons, l'imagerie par diffraction cohérente permet de reconstruire la transmittance complexe d'objets isolés avec une résolution spatiale limitée en théorie uniquement par la longueur d'onde d'illumination. Expérimentalement, on éclaire un objet unique (au contraire des motifs répétés un grand nombre de fois en cristallographie) et isolé (c'est-à-dire entouré d'une zone opaque) par une onde monochromatique cohérente spatialement et on enregistre en champ lointain la figure de diffraction ainsi créée (figure 1). Dans ce régime, appelé régime de Fraunhofer, le champ diffracté en champ lointain est égal à la transformée de Fourier de la transmittance complexe de l'objet. Cependant, les détecteurs utilisés (caméra CCD ou détecteur à pixels hybrides) ne sont sensibles qu'à l'intensité du champ. L'information sur la phase de cette transformée de Fourier est donc perdue, et une transformation inverse directe ne peut fournir l'image de l'objet. Aussi diverses techniques ont-elles été développées afin de retrouver cette image à l'aide d'algorithmes ad hoc. Nous utilisons au CEA un algorithme itératif de reconstruction de la phase. Dans cette approche, le fait d'imager un objet isolé est crucial pour plusieurs raisons. D'une part, ceci signifie que l'objet reconstruit a une extension spatiale finie, incluse dans un support entouré de «zéros». De plus, dans ces conditions, la figure de diffraction est continue (et non constituée de pics de Bragg discrets comme en diffraction X standard). II est alors possible de la sur-échantillonner (par rapport au critère de Nyquist) par un dimensionnement judicieux des paramètres de l'expérience.

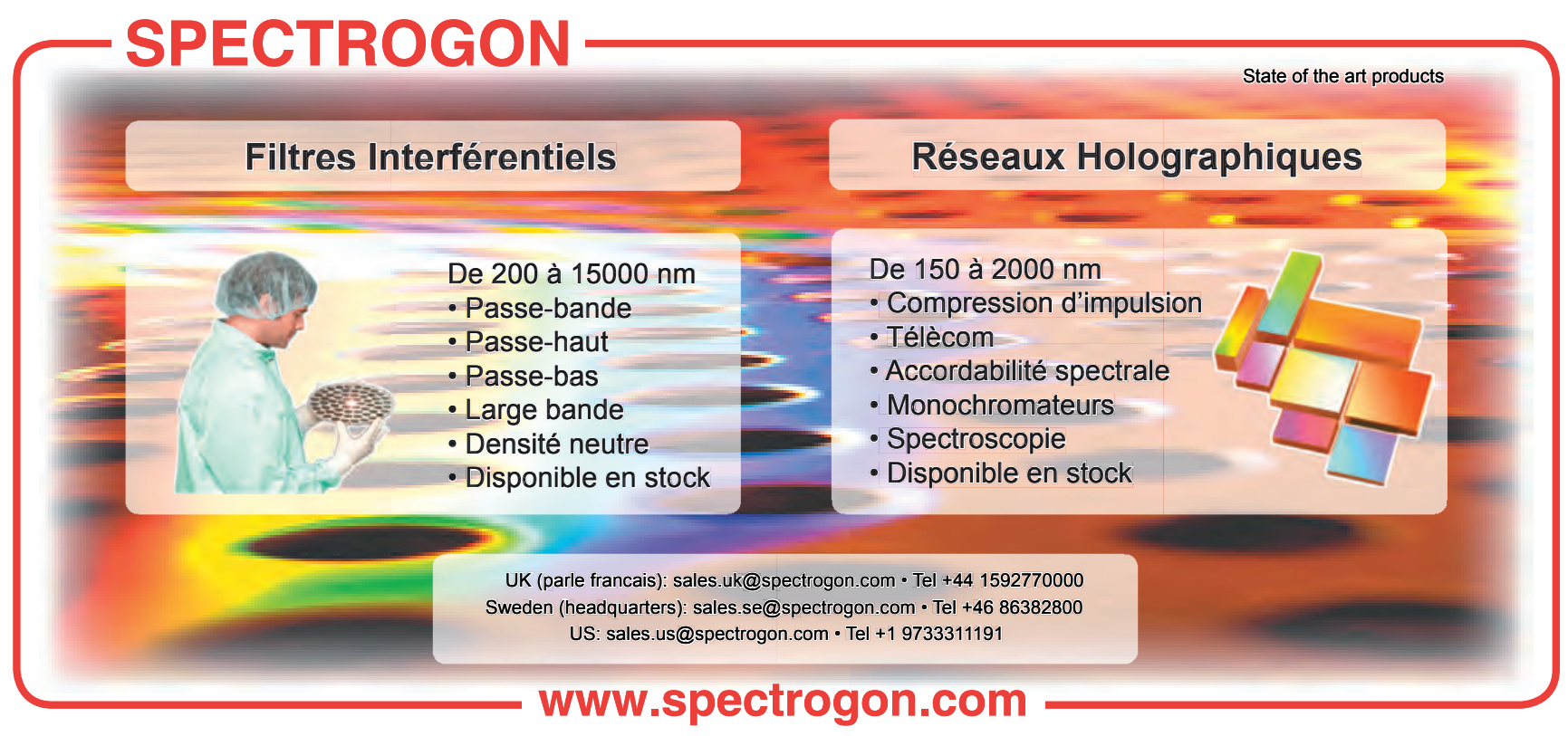




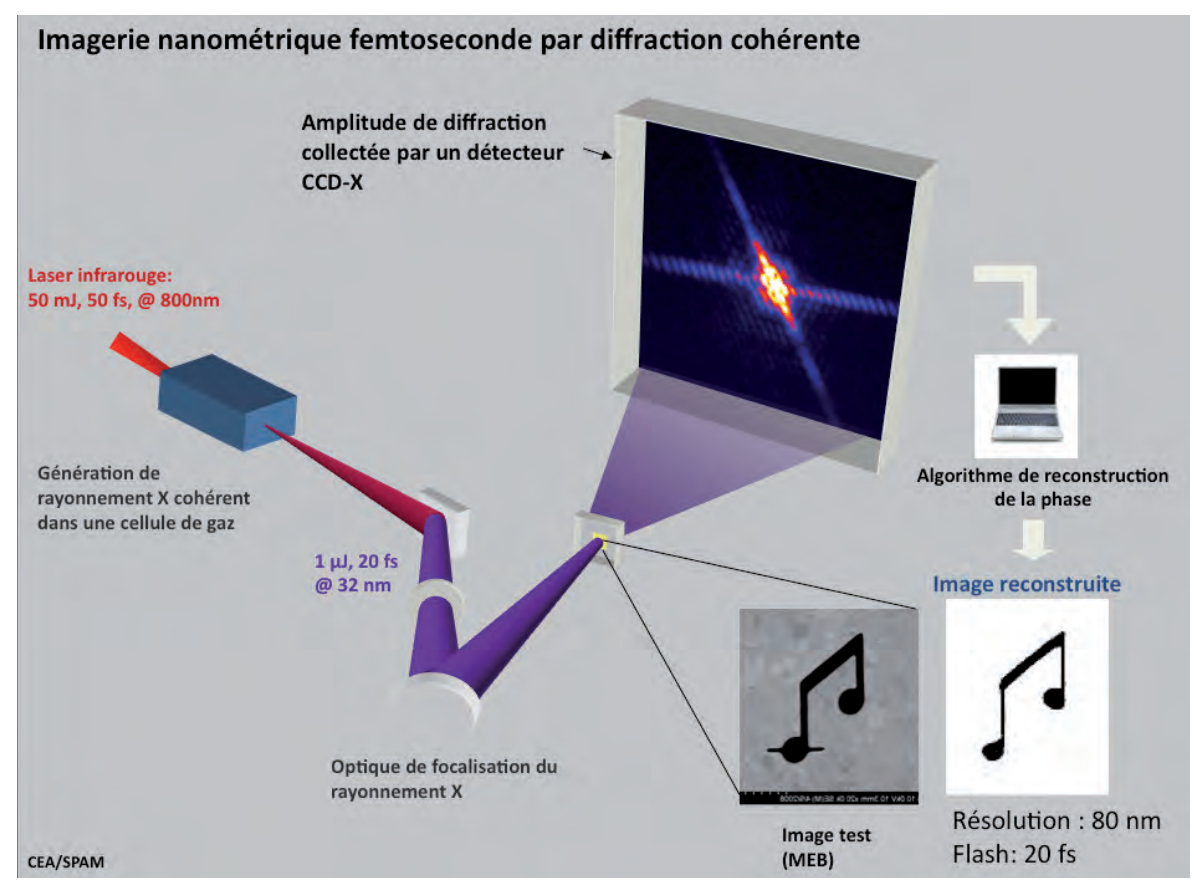

Figure 1. Schéma de l'expérience d'imagerie par diffraction cohérente réalisée au CEA.

Récemment, sur le laser à électrons libres $X$ durs de Stanford, une équipe internationale a réussi à imager un nano-cristal de complexes de protéines, se rapprochant ainsi d'une résolution spatiale atomique (Chapman et al., 2011).

\section{Holographie X}

La résolution spatiale que l'on peut obtenir par les techniques de reconstruction itérative est fortement dépendante de la qualité des données expérimentales. Si la résolution théorique est donnée par la longueur d'onde utilisée, dans la pratique elle dépend de l'ouverture angulaire du signal enregistré et est donc généralement limitée par le bruit. De plus, si la qualité des données n'est pas suffisante, les algorithmes de reconstruction peuvent ne pas converger ou laisser subsister un doute sur la fidélité de la reconstruction. Les techniques d'holographie, grâce à une reconstruction directe (et sans problème de convergence dans le processus de reconstruction de la phase), permettent de lever cette ambiguïté. Parmi les différentes formes d'holographies disponibles, I'holographie par transformée de Fourier est la plus utilisée actuellement. Il s'agit de placer à proximité de l'échantillon une référence (un petit trou par exemple, comme sur la figure $2 b$ ) qui va diffracter une onde sphérique.

Au niveau du détecteur, cette onde interfère avec l'onde diffractée par l'échantillon. L'amplitude et la phase de la transformée de Fourier de l'objet sont ainsi directement encodées

Figure 2. Étude de trois objets test sur la ligne laserharmoniques du CEA Saclay en régime simple tir. La colonne de gauche présente une image MEB de l'échantillon (une membrane de $\mathrm{Si}_{3} \mathrm{~N}_{4}$ avec un dépôt $\mathrm{d}^{\prime}$ or de $100 \mathrm{~nm}$ environ gravée au faisceau d'ions focalisés) ; la colonne du milieu l'image de diffraction enregistrée par la caméra CCD en champ lointain; et la colonne de droite les images obtenues par trois techniques différentes, (a) : algorithme itératif de reconstruction de la phase, (b) : holographie par transformée de Fourier, (c) : holographie avec références étendues. dans cette figure d'interférence (appelée hologramme). Une simple transformation deFourier inverse del'hologramme permet alors de reconstruire la transmittance de l'échantillon.

La résolution spatiale est dans ce cas aussi limitée par le signal aux larges angles de diffraction, mais dépend de plus de la taille de la référence : plus la référence est petite, meilleure sera la résolution théorique, au détriment cependant de la qualité du signal, ce qui limite en pratique l'utilisation de l'holographie. 
Pour contourner cette difficulté, il est possible par exemple de combiner les deux techniques précédentes : I'utilisation d'une référence trop large donne une reconstruction de l'échantillon de faible résolution, laquelle est utilisée comme support initial pour un algorithme itératif. Une autre technique, dérivée de I'holographie par transformée de Fourier, utilise des références étendues combinées à des opérateurs différentiels adaptés à la forme de ces références. L'idée de cette technique, appelée HERALDO (pour Holography with Extended Reference by Autocorrelation Linear Differential Operation) (Guizar-Sicairos et al., 2007), consiste à utiliser une opération mathématique simple (une ou plusieurs dérivations) afin d'obtenir un équivalent de références ponctuelles à partir de références plus larges, telles que des fentes ou des carrés (figures $2 c$ et 3). On obtient ainsi un hologramme présentant des interférences de meilleure visibilité aux grands angles tout en conservant une bonne résolution

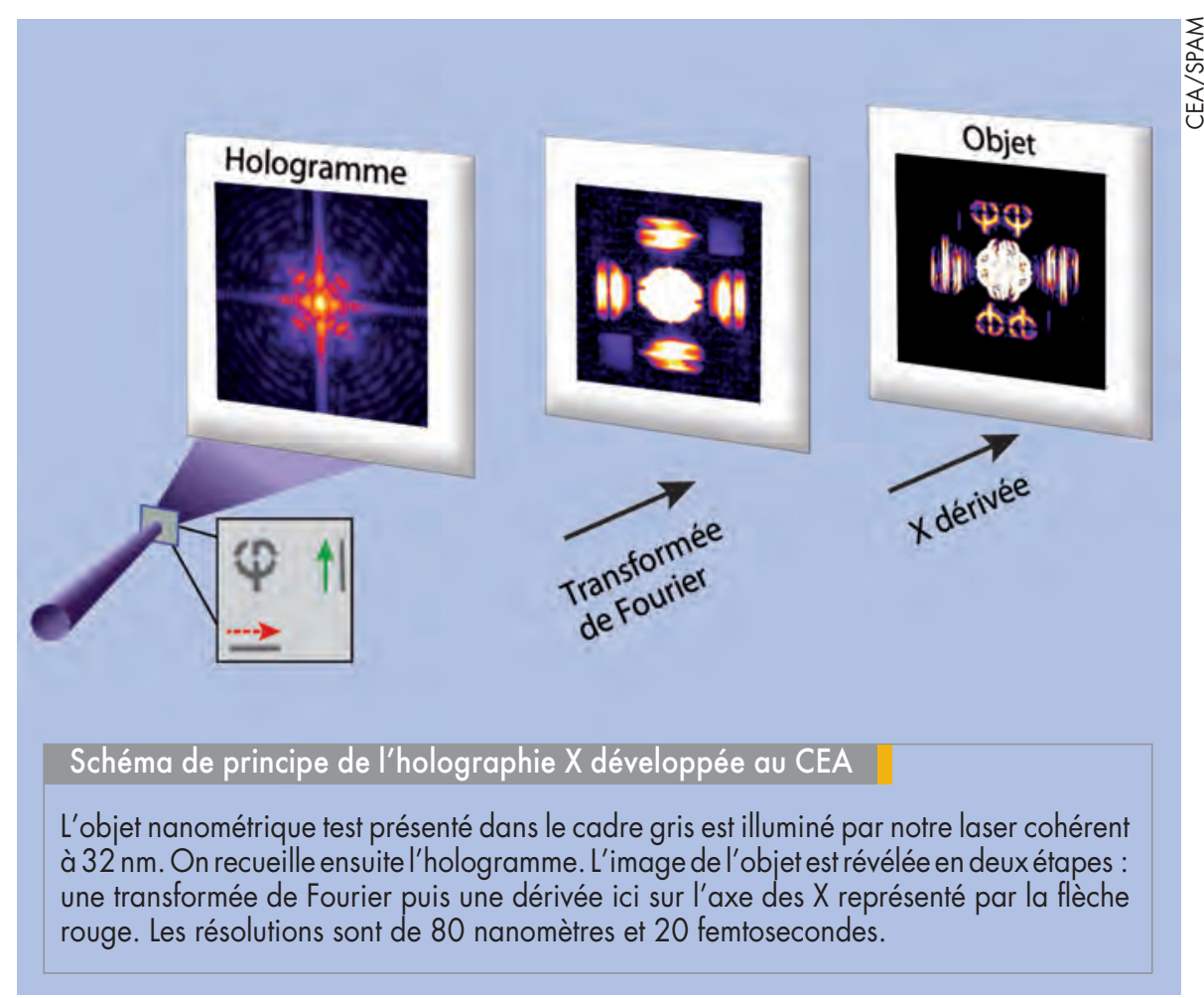

Figure 3. Principe de l'holographie X par transformée de Fourier et opérateur différentiel.

\section{High Resolution Microfocus X-ray Source L10711-02}

La Source à Rayons X L10711-02 de type "Nano-foyer" propose une taille de spot descendant à $0,25 \mu \mathrm{m}$. C'est la solution idéale pour un grand nombre d'applications de contrôle non destructif nécessitant une très bonne résolution, y compris en nano tomographie, inspection de PCB et plus encore.

Spécifications:

- Taille de spot de $1 \mu \mathrm{m}$ à $0,25 \mu \mathrm{m}$

- Tension de tube de $20 \mathrm{kV}$ à $160 \mathrm{kV}$

- Distance focale de $0,5 \mathrm{~mm}$

- Générateur "Haute tension" intégré

- Fonction "Auto alignement"

Applications:

- Micro et Nano tomographie

- Inspection de PCB

- Biologie

- Cristallographie 


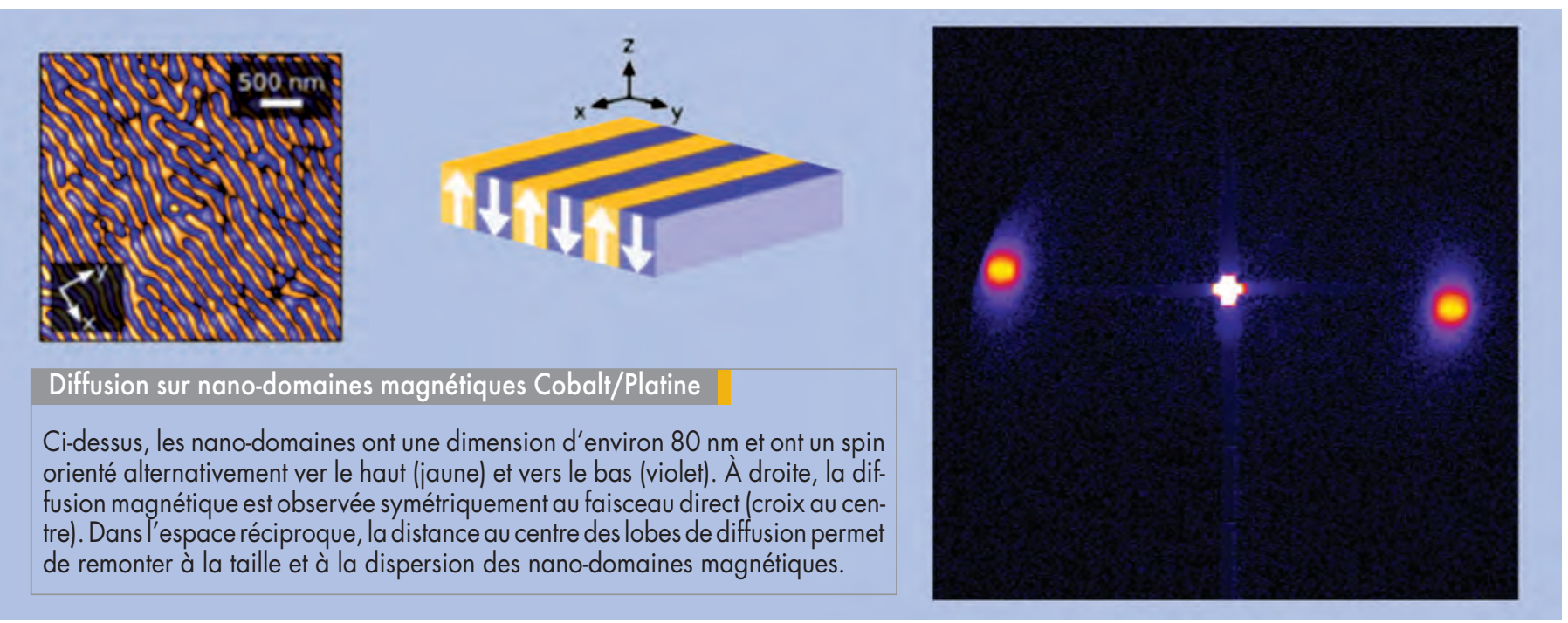

Figure 4. Figures de diffusion de nano-domaines magnétiques cobalt/platine. Ces mesures ont été effectuées près du seuil $\mathrm{M}$ du cobalt à 60 eV afin d'obtenir un contraste de phase.

spatiale. Nous avons pu démontrer que pour nos conditions expérimentales, la technique HERALDO permettait de reconstruire l'image d'un échantillon test avec une résolution de l'ordre de $100 \mathrm{~nm}$ (pour une longueur d'onde d'illumination de $32 \mathrm{~nm}$ ) là où, pour un même objet, les algorithmes itératifs ne convergeaient pas. Enfin, un avantage non négligeable des techniques d'holographie est qu'elles permettent une analyse immédiate des résultats. Les résolutions obtenues en simple tir laser sont de l'ordre de $100 \mathrm{~nm}$ sur un flash de lumière de $20 \mathrm{fs}$ (Gauthier et al., 2010).

\section{Imagerie nanométrique par flash femtoseconde et applications}

Quels sont les apports de l'imagerie par diffraction cohérente par rapport aux autres techniques d'imagerie existantes (microscopie électronique, microscopie $\mathrm{X}$ à balayage...) ? Son avantage principal tient en la possibilité d'obtenir une image d'un échantillon grâce à une unique impulsion sonde ultrabrève. En effet, il n'est pas nécessaire de balayer l'objet, et il existe des sources de rayons $X$ assez intenses pour fournir un signal suffisant sans avoir à accumuler sur de nombreuses impulsions successives. Ceci est utile pour deux raisons. Tout d'abord les rayons $X$ intenses peuvent induire des dommages aux échantillons, surtout biologiques, voire même leur destruction. Si les impulsions sondes ont une durée femtoseconde, la figure de diffraction sera enregistrée avant que l'objet ne se soit dégradé. De plus, certaines études dynamiques imposent l'utilisation de tirs uniques. C'est le cas par exemple lorsqu'il n'est pas possible de reproduire un échantillon à l'identique pour des études sur laser à électrons libres, lesquels détruisent les objets à chaque tir. Le problème se pose aussi en cas d'étude de phénomènes non reproductibles pour lesquels il est impossible d'accumuler sur plusieurs tirs consécutifs.

Ce type d'étude était jusqu'à il y a peu uniquement possible sur des lasers à électrons libres. À ce jour, la seule étude en imagerie nanométrique a été réalisée sur le laser à électrons libres UV-X FLASH à Hambourg en Allemagne avec une résolution picoseconde (étude de l'ablation d'un échantillon par laser) (Barty et al., 2008). Cependant le nombre d'installations ouvertes aux utilisateurs est limité, et le temps de faisceau disponible rare. Ces études sont maintenant possibles sur la ligne de lumière laser-harmoniques $X$ cohérents du CEA Saclay. Les premières expériences de mesure de dynamiques ultrarapides sont actuellement en cours au CEA. Un exemple concerne l'étude de la désaimantation d'échantillons magnétiques sous l'action d'un champ laser infrarouge ultrabref : ce champ laser peut faire disparaître temporairement l'aimantation ou inverser sa direction. L'une des questions qui se posent alors concerne la dynamique de ces phénomènes, question reliée par exemple à la vitesse ultime de lecture/enregistrement pour des dispositifs de mémoires magnétiques par laser. Afin d'y répondre, nous étudions la désaimantation d'échantillons magnétiques formés de couches de cobalt et de palladium superposées. Les nano-domaines magnétiques possèdent une aimantation perpendiculaire à leur surface et de sens alternés. Un champ infrarouge intense fait disparaître l'aimantation en un temps de l'ordre de 100 fs. En fonction de la fluence du laser de pompe, la structuration magnétique peut ensuite réapparaître à l'identique, ou au contraire être fortement modifiée. II a déjà été démontré sur synchrotron et laser à électrons libres qu'il était possible de visualiser ces domaines par les techniques d'imagerie cohérente en régime statique. Cependant, les études dynamiques en régime de forte fluence nécessitent une imagerie simple tir ainsi qu'une bonne synchronisation entre le laser de pompe et le rayonnement XUV. Dans une étape préliminaire, nous avons montré que notre ligne 
de lumière permettait d'obtenir des images de diffusion magnétique de ces échantillons en régime de tir unique et de déduire de ces images des informations sur la distribution spatiale des domaines (figure 4) (Ducousso et al., 2012). L'étape suivante consiste à inverser ces figures de diffraction afin d'imager les échantillons. Les études résolues en temps sont en cours. Le but est d'observer la démagnétisation ultra-rapide (échelle de temps typique $100 \mathrm{fs}$ ) des nano-domaines.

\section{Discussion et conclusion}

Nous avons présenté les applications en imagerie nanométrique femtoseconde de la source laser-harmoniques du CEA Saclay. Les efforts récents ont permis non seulement de générer un flux de photons importants jusqu'au microjoule (environ $10^{11}$ photons à $32 \mathrm{~nm}$ ) par impulsion à $20 \mathrm{~Hz}$, mais aussi d'obtenir d'excellentes qualités spatiales. Une géométrie en longue focale permet de "coupler» une énergie laser plus élevée avec le milieu en augmentant la dimension transverse de la source. Les deux techniques d'imagerie sans lentille ont été réalisées en simple tir: la diffraction cohérente est basée sur un algorithme de reconstruction de la phase alors que I'holographie par transformée de Fourier avec référence étendue permet une reconstruction directe. La résolution de la reconstruction des images est d'environ $100 \mathrm{~nm}$ en simple tir ( $20 \mathrm{fs}$ de résolution temporelle) et permet ainsi d'envisager des applications de type pompe sonde. L'imagerie par diffraction cohérente présente de nombreux avantages. Contrairement à l'holographie, elle n'est pas limitée par la qualité de gravure. Potentiellement, la résolution en imagerie par diffraction cohérente peut être très proche de la longueur d'onde (nous avons obtenu au mieux $2 \lambda$ dans notre expérience). Dans la pratique, la résolution de l'image va être surtout limitée par le signal, la cohérence et le front d'onde du faisceau. Dans certains cas, ces différentes limitations empêchent même la convergence de l'algorithme et donc de reconstruire une image. Ceci peut être encore plus accentué dans une configuration de type pompe-sonde où l'amplitude et la phase d'un objet/processus peuvent évoluer dans l'espace et le temps. C'est la raison pour laquelle nous avons développé une configuration holographique permettant une reconstruction directe, nonitérative, en deuxétapes (dérivée et TF). La technique HERALDO $a$ un très fort potentiel. Elle est moins limitée par le signal émis par la référence (trou de référence étendu : fente, carré, triangle) que dans I'holographie par transformée standard (trou de référence sphérique). La qualité des images obtenue est remarquable. Nous avons aussi obtenu de très bonnes résolutions, proche de $2 \lambda$ en 10 tirs et de $3 \lambda$ en simple tir. La technique HERALDO s'est montrée aussi très robuste par rapport aux défauts de gravure de la référence et à la non-uniformité de l'illumination de la référence.

Finalement, ces nouvelles techniques d'imagerie permettent d'obtenir des résolutions spatio-temporelles uniques, nanométrique et femtoseconde. L'imagerie en simple tir exposée ici est cruciale notamment parce qu'elle permet de capturer une image avant que des dommages ou des dégradations de l'objet n'interviennent. C'est aussi le seul moyen d'imager des processus non-reproductibles rencontrés par exemple dans certaines transitions de phase à l'échelle mésoscopique ou dans l'étude de renversement de spin de nanodomaines magnétiques présentée ci-dessus, ou bien encore dans l'imagerie de réaction d'isomérisation de complexes nanométriques à base d'azobenzene que nous étudions actuellement dans le cadre d'un projet franco-canadien.

\section{Références}

Barty et al., Nature Photonics 2, 415 (2008). Chapman et al., Nature Physics 2, 839 (2006). Chapman et al., Nature 470, 70 (2011).

Ducousso et al., soumis à Phys. Rev. Lett. (2012). Gauthier et al., Phys. Rev. Lett. 105, 093901 (2010).

Guizar-Sicairos et al., Optics Express 154, 17592 (2007).

McNulty et al., Science 256, 1009 (1992).

Miao et al., Nature 400, 342 (1999).

Ravasio et al., Phys. Rev. Lett. 103, 028104 (2009).

Seibert et al., Nature 470, 78-81 (2011).

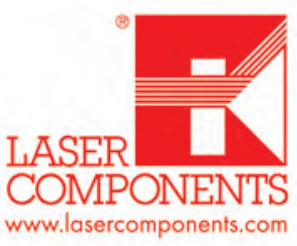

\section{A la \\ recherche du} meilleur nez?

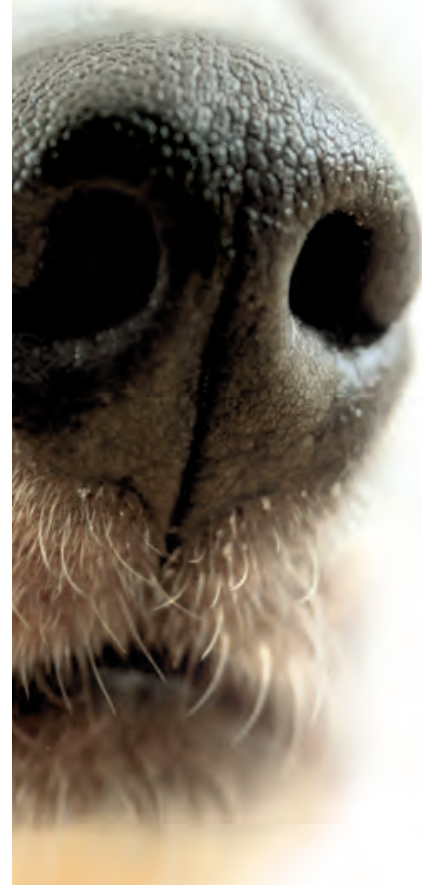

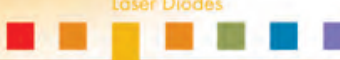

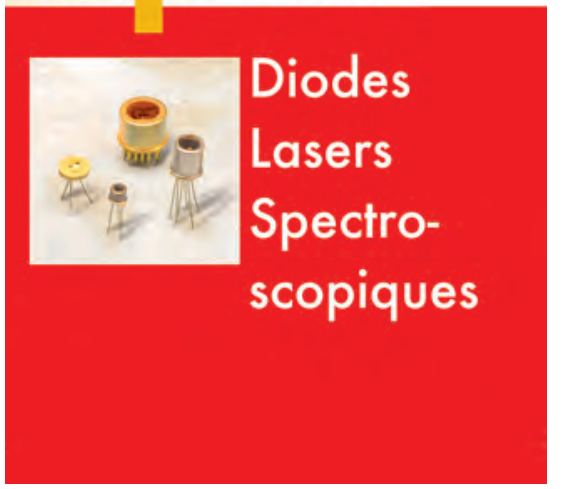

